observations were carried out in the Himalayas in a region that has extremely high thunderstorm activity (about 30 lightning strokes per day). Since the late 1990s, many other studies have also claimed statistically significant detections of thunderstorm-produced neutrons from all over the world ${ }^{7-10}$. However, the detectors could not distinguish neutrons from other particles such as electrons and $\gamma$-ray photons - all three would produce similar electriccurrent pulses in the detectors ${ }^{11}$.

It was initially thought that thunderstorminduced neutrons were produced in a nuclear reaction in which two nuclei of the hydrogen isotope deuterium fuse in the plasma created by lightning to form a helium nucleus and a neutron. However, it was later shown that the physical conditions in such a plasma do not allow this reaction to occur ${ }^{12}$.

Instead, the avalanche of high-energy electrons produced in a thundercloud emits X-ray and $\gamma$-ray photons. Since the late 1980s, these photons have been detected on the ground, by aircraft flying inside thunderclouds, and by artificial satellites in near space (about 500 kilometres above Earth's surface) ${ }^{13}$. The photons have energies of up to hundreds of megaelectronvolts $(\mathrm{MeV})$.

High-energy electrons, and $\gamma$-rays that have energies larger than about $10 \mathrm{MeV}$, can knock out neutrons from atmospheric nitrogen-14 and oxygen-16 nuclei - by electrodisintegration in the case of electrons and photonuclear reactions in the case of $\gamma$-rays ${ }^{11,12}$. Although the ability of thunderstorms to produce neutrons through photonuclear reactions has been demonstrated using computer simulations ${ }^{11,13}$, direct experimental evidence has been absent.

Rather than focusing on the neutrons, Enoto and colleagues considered the other products of the photonuclear reactions involving nitrogen-14 and oxygen-16: namely, unstable nitrogen-13 and oxygen-15 isotopes (Fig. 1). These isotopes decay after a few minutes into stable carbon-13 and nitrogen-15 nuclei through the emission of a neutrino and a positron - the antiparticle of the electron. Finally, the positron annihilates with an electron of an atmospheric molecule to produce a pair of $\gamma$-rays.

Because both positrons and electrons have masses of $0.511 \mathrm{MeV}$ (expressed in energy units), each emitted $\gamma$-ray has an energy of $0.511 \mathrm{MeV}$. Therefore, to confirm the existence of these photonuclear reactions, the authors simply needed to identify a line at this energy in the wide energy spectrum of all $\gamma$-rays.

To this end, Enoto et al. carried out groundbased observations of $\gamma$-ray emission from low winter thunderclouds above the coast of the Sea of Japan. On 6 February 2017, they detected an intense $\gamma$-ray flash that lasted for less than 1 millisecond, which they associated with a lightning stroke. After the initial $\gamma$-ray flash, the authors observed a prolonged $\gamma$-ray line at an energy of $0.511 \mathrm{MeV}$ that lasted for about a minute (see Fig. 4 in the paper ${ }^{2}$ ). This line is a conclusive indication of electronpositron annihilation, and represents unequivocal evidence that photonuclear reactions can be triggered by thunderstorms.

Enoto and colleagues' discovery is important because it unveils a previously unknown natural source of isotopes in the atmosphere, in addition to the irradiation of Earth by cosmic rays. These isotopes include nitrogen-15, carbon-13 and carbon-14, the last of which is widely used in the dating of archaeological artefacts and artworks. In fact, the contribution of thunderstorms to Earth's carbon-14 abundance could be comparable in some regions to that of cosmic irradiation ${ }^{14}$. Future studies should check whether thunderstorms produce other isotopes (such as those of hydrogen, helium and beryllium).

Thunderstorm-induced nuclear reactions could occur in the atmospheres of other planets, such as Jupiter and Venus, and might therefore contribute to the isotopic composition of these atmospheres. However, determining the magnitude of this contribution will require detailed observations of $\gamma$-rays and neutrons from thunderstorms on these planets. Another implication of Enoto and colleagues' discovery is that the neutrons are formed outside the plasma created by lightning. This suggests that these neutrons cannot provide information about the plasma, in contrast to expectations ${ }^{15}$.

Leonid Babich is at the Russian Federal

Nuclear Center - All-Russian Research

Institute of Experimental Physics

(RFNC-VNIIEF), Sarov, Nizhni Novgorod

region, 607185 Russia.

e-mail:leonid.babich52@gmail.com

1. Wilson, C. T. R. Proc. Cambridge Phil. Soc. 22, 534-538 (1925).

2. Enoto, T. et al. Nature 551, 481-484 (2017).

3. Chadwick, J. Nature 129, 312 (1932).

4. Gurevich, A. V., Milikh, G. M. \& Roussel-Dupre, R. Phys. Lett. A 165, 463-468 (1992).

5. Eddington, A. S. Nature 117, 25-32 (1926).

6. Shah, G. N., Razdan, H., Bhat, C. L. \& Ali, G. M. Nature 313, 773-775 (1985).

7. Chilingarian, A. et al. Phys. Rev. D 82, 043009 (2010).

8. Gurevich, A. V. et al. Phys. Rev. Lett. 108, 125001

(2012).

9. Tsuchiya, H. et al. Phys. Rev. D 85, 092006 (2012).

10.Ishtiaq, P. M., Mufti, S., Darzi, M. A., Mir, T. A. \&

Shah, G. N. J. Geophys. Res. Atmos. 121, 692-703 (2016).

11. Babich, L. P., Bochkov, E. I., Kutsyk, I. M. \& Zalyalov, A. N. JETP Lett. 97, 291-296 (2013).

12.Babich, L. P. JETP Lett. 84, 285-288 (2006).

13. Dwyer, J. R., Smith, D. M. \& Cummer, S. A. Space Sci. Rev. 173, 133-196 (2012)

14.Babich, L. P. Geophys. Res. Lett. 44, http://dx.doi. org/10.1002/2017GL075131 (2017).

15.Fleisher, R. L., Plumer, J. A. \& Crouch, K. J. Geophys. Res. 79, 5013-5017 (1974).

\title{
How T cells spot tumour cells
}

Immunotherapy can reawaken T cells to destroy tumour cells. Modelling of
tumour and T-cell interactions suggests why certain tumour cells are targeted
and improves predictions of immunotherapy outcome. SEE LETTERS P.512 \& P.517

\section{SIRANUSH SARKIZOVA \& NIR HACOHEN}

$\mathrm{T}$ The $\mathrm{T}$ cells of the immune system have a key role in the identification and elimination of cells that pose a threat to the body, such as infected cells and cancer cells. Two papers by Balachandran et al. ${ }^{1}$ (page 512) and Łuksza et al. ${ }^{2}$ (page 517), which have many authors in common, propose a framework to assess how effectively tumours can be detected by $\mathrm{T}$ cells - a tumour property known as immunogenicity. The authors demonstrate that their models for assigning tumour-immunogenicity scores can be used to predict clinical responses to a type of cancer immunotherapy called checkpoint blockade.

Most cells in the body present peptide fragments known as antigens on their cell surface, which are generated from intracellular proteins. Each peptide is bound in a complex with a specialized receptor called an MHC class I protein (HLA class I in humans). T cells known as cytotoxic $\mathrm{T}$ cells police the body in search of cells displaying specific antigens, especially antigens from infectious organisms, or in the case of cancer, antigens known as neoantigens that have arisen as a result of a mutation (Fig. 1). If the T-cell receptor (TCR) of a cytotoxic $\mathrm{T}$ cell recognizes and binds an antigen that is not normally present, the $\mathrm{T}$ cell will often unleash an attack that kills the cell displaying that antigen. TCRs are highly variable and have slightly different antigen-binding regions, enabling the immune system to recognize millions of antigens ${ }^{3}$. Antigen binding to MHC proteins and TCR recognition of antigen-MHC complexes are key determinants of an immune response.

Tumour cells often fight back against this 
immune-system surveillance by hijacking the natural mechanisms that dampen immune responses, which are normally intended to block autoimmmune attacks against healthy tissue. Checkpoint-blockade therapies can block these immuno-inhibitory signals, such as those generated by the 'checkpoint' PD-L1 protein ${ }^{4}$. However, only a subset of tumours treated with such therapies regress. Therefore, approaches are needed to identify the tumours that are most likely to respond to immunotherapy.

Current ways of predicting the effectiveness of checkpoint-blockade therapy rely on measuring the level of PD-L1 protein expressed by tumour cells, counting the number of T cells in a tumour, and estimating the number of different neoantigens that a tumour contains ${ }^{5}$. The work by Łuksza and Balachandran and their respective colleagues offers a new type of integrated model to predict whether a tumour will be attacked by T cells, a characteristic that they refer to as tumour fitness (low fitness being associated with a strong immune response against the tumour).

The authors calculate tumour fitness by assessing the immunogenicity of the neoantigens that a tumour contains. To estimate the immunogenicity of each neoantigen, the authors first considered how tightly each patient's MHC protein binds to each given neoantigen compared with the non-mutant version of the antigen, and second, they assigned a score for the likelihood of a neoantigen-MHC complex being recognized by a TCR. Although MHC shape can vary depending on the version of MHC protein present, computational algorithms can accurately predict the affinity of a given antigen for any version of MHC protein encoded in a patient's genome.

However, predicting which antigens are more likely than others to be recognized by TCRs remains a challenge. To tackle this problem, both groups of authors make the simplifying assumption that neoantigens are more likely to be immunogenic if they resemble infectious-disease-associated antigens that are known to stimulate T cells and therefore might have a higher probability of being recognized as 'non-self'.

In both studies, the authors calculated the fitness of each individual tumour on the basis of the combined fitnesses of the subpopulations of tumour cells, known as clones, that contain different mutations. Each clone in the tumour was represented by a score for its neoantigen that was most likely to bind MHC and be recognized by a TCR. Łuksza and colleagues validated their model in an analysis of three groups of individuals diagnosed with cancer (two groups with melanoma and one group with non-small-cell lung cancer) and being treated with checkpoint-blockade therapy. The tumours predicted by their model to have lower fitness were indeed associated with longer patient-survival times. Furthermore,


Figure 1 | Predicting whether cancer mutations will trigger an immune response. a, Fragments of intracellular proteins, known as antigens, bind to MHC receptor proteins and might be recognized by T-cell receptor (TCR) proteins and trigger an immune response. Tumour cells contain mutated antigens called neoantigens that are not normally present in the body (expression of different neoantigens indicated by different shades of red). Łuksza and colleagues' model ${ }^{2}$ assigns a score for how likely it is that a particular neoantigen will elicit an immune response on the basis of how well it binds to the MHC protein and the likelihood of it being recognized by a TCR. A high score is given to a neoantigen predicted to elicit a strong T-cell response (dark blue) and a low score given to a neoantigen predicted to elicit a weak T-cell response (light blue) or no T-cell response. The neoantigen scores for each cellular clone were then considered together to predict how well a tumour will be controlled by the immune system. $\mathbf{b}$, Balachandran et al. ${ }^{1}$ demonstrate that this type of model could be used to distinguish between a group of individuals who died of pancreatic cancer within a median time after diagnosis of more than eight years (red) or less than one year (blue). (Panel based on part of Fig. $2 \mathrm{~b}$ of ref. 1.)

they demonstrated that the predictive power of their full model is superior to partial versions that used only some of the scoring criteria.

Balachandran and colleagues demonstrated that this type of modelling approach could distinguish between long-term and short-term survivors after a diagnosis of pancreatic cancer. An alternative model that they tested, which assessed the number of different neoantigens, could not. Furthermore, when blood samples from patients were analysed, Balachandran et al. found that, compared with short-term survivors, the long-term survivors had a higher frequency of neoantigens generated from mutations in the gene MUC16 and had T-cell responses against mutant MUC16. The authors suggest that MUC16 mutations generate neoantigens that are key antitumour targets of the immune system.

The tumour-fitness model is a mathematical model in which several parameters need to be set. These parameters relate to the timescale of response to therapy and the assessment of the probability that a neoantigen will be recognized by a TCR. To determine the numerical values to use for these parameters, both groups varied these parameter values so that the model would best match the observed patient-survival data.

Łuksza and colleagues employed an approach in which the parameters determined using data from one group of patients were used to predict survival of an independent group of patients. This is a commonly accepted way of avoiding a problem known as 'over-fitting, in which a model works only for a particular data set. Balachandran and colleagues determined the parameter values and predicted outcomes using the same group of patients, but argued that similar values were obtained when subsamples of the data were used for this parameter-setting purpose. The need to set the model parameters for each group of patients raises the question of how predictive the model will be for any given patient, considering the unique characteristics of their individual tumour type and the particular type of immunotherapy used.

Many other factors - in addition to affinity of antigen for MHC proteins and TCR antigen recognition - can influence the potency of neoantigens or correlate with checkpointblockade effectiveness. Thus, it would be desirable to be able to extend the model easily. Indeed, Łuksza and colleagues demonstrate the incorporation of additional parameters, such as the expression of cytotoxic genes in the tumour microenvironment, which is associated with immune-cell targeting of the tumour, and show that this addition improves the model's predictive power. In the same spirit, a superior model could be achieved by taking yet more factors into account, such as biases in the protein-degradation process that generates antigens, or the level of neoantigen expression.

It remains possible that the similarity of a neoantigen to non-microbial antigens, such as antigens associated with autoimmunity, could also be used to predict immunogenicity and patient survival. Thus, more research is needed to pinpoint the basis of TCR recognition of antigens. Although many factors must be considered in the prediction of effective antitumour immune responses in a given patient 
(including non-mutant antigens, which can sometimes trigger an anticancer response), neoantigens are emerging as crucial targets that $\mathrm{T}$ cells can use to detect and destroy cancer cells, and represent important targets for immunotherapy ${ }^{6-8}$.

Siranush Sarkizova and Nir Hacohen are at the Center for Cancer Research,
Massachusetts General Hospital,

Boston, Massachusetts 02114, USA, and at the Broad Institute of MIT and Harvard, Cambridge, Massachusetts. S.S. is also in the Department of Biomedical Informatics, Harvard Medical School, Boston. e-mail:nhacohen@broadinstitute.org

1. Balachandran, V. P. et al. Nature 551, 512-516 (2017).
2. Łuksza, M. et al. Nature $\mathbf{5 5 1 , 5 1 7 - 5 2 0 ~ ( 2 0 1 7 ) . ~}$

3. Robins, H. Curr. Opin. Immunol. 25, 646-652 (2013).

4. Sharma, P. \& Allison, J. P. Science $\mathbf{3 4 8 , 5 6 - 6 1}$ (2015).

5. Schumacher, T. N. \& Hacohen, N. Curr. Opin. Immunol. 41, 98-103 (2016).

6. Carreno, B. M. et al. Science 348, 803-808 (2015).

7. Ott, P. A. et al. Nature 547, 217-221 (2017).

8. Sahin, U. et al. Nature 547, 222-226 (2017).

\title{
Crowdsourcing Earth's microbes
}

\begin{abstract}
A large-scale study has been assessing microbial diversity by analysing DNA sequences from samples submitted by scientists around the globe. The initial results are now being used to create an open-access resource. SEE ARTICLE P.457
\end{abstract}

\section{JEROEN RAES}

A simple, though dauntingly ambitious idea of sampling the microbial genetic diversity across Earth is the driving force behind the Earth Microbiome Project. On page 457, Thompson et al. ${ }^{1}$ report the results of this experimental tour de force.

The project began life at a meeting in Snowbird, Utah, in 2010 at which a group of scientists from a wide range of disciplines discussed the goals, challenges and practicalities of such an enterprise ${ }^{2}$. Seven years on, the authors now report the microbial compositional profiles of a whopping 27,751 samples from 97 independent studies, providing insights into the diversity of microorganisms - from the bacterial and archaeal domains - in a wide range of geographic and environmental ecosystems, both terrestrial and aquatic. From these samples, Thompson and colleagues generated 2.2 billion DNA sequence reads of a highly variable region of a universally evolutionarily conserved gene called 16S rRNA, which encodes a component of the ribosome (the cell's protein-synthesis machinery).

The remarkable nature of this study lies not only in its scale and in the breadth of the environmental samples analysed (Fig. 1), but also in its methodology. The project involved a massive, global crowdsourcing effort in which scientists raided their collection freezers for samples to share with the project.

The approach was straightforward. A call was made for scientists to contribute well-preserved environmental samples collected during specific research projects, and the Earth Microbiome Project offered to sequence the DNA of the 16S rRNA gene in the microbial samples and to make the data available as open access.

This project is a prime example of a trend in the adoption of scientific approaches involving widespread engagement, in which the ease of electronic communication and the power of social media are harnessed to generate useful resources. In the same spirit, in the Polymath Project, mathematicians collaborate to tackle challenging mathematical problems.



Figure 1 | Environmental sources of microbial samples analysed by the Earth Microbiome Project. Thompson et al. ${ }^{1}$ report data generated from their work in a project to assess microbial diversity across Earth. Scientists from 43 countries sent the project's authors 27,751 DNA samples of free-living and host-associated microorganisms collected from various environments. After a quality-control step, the authors analysed part of the sequence of a gene called 16S rRNA in 23,828 of the samples.
Such approaches to generating crowdsourced experimental data usually work by first getting the project under way and obtaining funding later as the project gathers momentum, perhaps by crowdfunding. Examples of crowdsourced projects include those analysing bacteria in the human gut, such as the Flemish Gut Flora Project ${ }^{3}$, or the Personalized Nutrition Project ${ }^{4}$. Such studies contrast with conventional research collaborations that begin once a grant is obtained from a funding agency.

Challenges inevitably arise in the type of work conducted by Thompson et al., particularly from having to handle samples from many collection sites. A common frustration in microbial research is that sampling procedures, storage, transport conditions, DNA extraction and amplification protocols often result in a 'lab-of-origin' effect that makes it difficult to compare data generated by different research groups. To address this, the Earth Microbiome Project developed a range of protocols ${ }^{5}$ and standards for sample collection, DNA extraction, transport and the formatting of the associated auxiliary data (such as temperature or location), as well as data-analysis procedures. These protocols were used for the project itself, but have been rapidly adopted by the wider research community, and more than 2,000 papers have already been published that use them ${ }^{6}$. By having a single protocol for all samples, and running all analyses in one laboratory, Thompson and colleagues have tried to remove as many potential technical confounding factors as possible.

The results seem to confirm that they have succeeded, revealing that sample microbial profiles cluster by environment - those from a specific type of environment are more similar to each other than to those from other types of environment, irrespective of the research group that collected the sample. This approach also has a drawback, because a single DNAextraction protocol cannot be expected to perform equally well across the wide chemical and biological variability of the samples collected in this type of broad survey, and might be less effective than a targeted approach in which extraction protocols are optimized for the environment being sampled. Thompson and colleagues have favoured generalizability over sensitivity, a choice that can surely be defended in these circumstances.

Another limitation of the study is its lack of hypothesis-driven experimental design, 